\section{Gene-expression signatures predict survival in patients with DLBCL}

Cyclophosphamide, doxorubicin, vincristine and prednisone (CHOP) chemotherapy has been widely used for the treatment of patients with diffuse large B-cell lymphoma (DLBCL). The addition of rituximab to $\mathrm{CHOP}$ (R-CHOP) has increased survival rates of patients with DLBCL; however, because of the heterogeneity of the disease, whether rituximab benefits specific subgroups of patients is unclear.

The investigators of the Lymphoma/ Leukemia Molecular Profiling Project assessed whether the gene-expression profiles of patients with DLBCL who received $\mathrm{CHOP}$ ( $n=181$; training group) or R-CHOP $(n=233$; validation group) correlated with treatment. Three gene-expression signatures predicted survival in both groups, and also in an independent cohort of patients treated with $\mathrm{CHOP}$; the germinal-center B-cell and the stromal-1 signatures were associated with increased survival, while the stromal-2 signature was associated with decreased survival. The germinal-center B-cell signature genes were highly expressed in malignant cells in contrast to stromal-1 and stromal-2 signature genes, which were highly expressed in normal cells. Tumors with extracellular matrix deposition that were infiltrated by cells of the monocytic lineage were identified by high expression of the stromal-1 gene signature. The stromal-2 signature included genes encoding markers of endothelial cells and regulators of angiogenesis, and high relative expression of this signature correlated significantly with blood-vessel density $(P=0.021)$.

The results showed that two new gene signatures can predict survival of patients with DLBCL treated with CHOP or R-CHOP.

Original article Lenz G et al. (2008) Stromal gene signatures in large-B-cell lymphomas. N Engl J Med 359: 2313-2323

\section{Mohs surgery is superior to excision for recurrent facial BCC}

A randomized study from The Netherlands suggests that Mohs micrographic surgery is superior to surgical excision as treatment for recurrent basal-cell carcinoma (BCC) of the face.
In an intention-to-treat analysis with 5 years' follow-up, Mosterd et al. randomly assigned 374 patients with 408 primary BCCs, and 191 patients with 204 recurrent BCCs, to undergo either Mohs micrographic surgery or surgical excision. Full follow-up data were available for 251 patients with 271 primary BCCs and 137 patients with 146 recurrent BCCs. Four recurrences $(2.5 \%)$ of primary BCC after Mohs micrographic surgery occurred during follow-up, compared with seven recurrences $(4.1 \%)$ after surgical excision; the difference was not statistically significant. Twelve recurrences occurred in the patients with recurrent BCC: two $(2.4 \%)$ in those treated with Mohs micrographic surgery, and ten $(12.1 \%)$ in those who underwent surgical excision; this difference was significant $(P=0.015)$. The incremental cost-effectiveness ratios per recurrence avoided were $€ 23,454$ for primary BCC and $€ 3,171$ for recurrent BCC.

The authors conclude that Mohs micrographic surgery should be preferred to surgical excision for treatment of recurrent BCC. Surgical excision is probably preferable for most cases of primary BCC on the basis of costeffectiveness. However, for more-aggressive subtypes of facial primary BCC, because of the increased risk and the adverse cosmetic effect associated with repeated surgery, Mohs micrographic surgery is recommended.

\footnotetext{
Original article Mosterd K et al. (2008) Surgical excision versus Mohs' micrographic surgery for primary and recurrent basal-cell carcinoma of the face: a prospective randomised controlled trial with 5-years' follow-up. Lancet Oncol 9: 1149-1156
}

\section{Exon 11 KIT genotype improves outcome in imatinib treated GIST}

The KIT inhibitor, imatinib, is the standard treatment for patients with advanced gastrointestinal stromal tumor (GIST). Previous studies have shown, however, that treatment outcomes vary depending on the kinase genotype of the patient. Heinrich and colleagues investigated the relationship between imatinib dosing, KIT genotypes and clinical outcome in patients with GIST.

This prospective, phase III study included tumor samples from 428 patients who were treated with $400 \mathrm{mg}$ or $800 \mathrm{mg}$ per day of imatinib. As shown in previous studies, the most 